\title{
Mosaic length and finite interaction-range effects in a one dimensional random energy model
}

\author{
S. Franz,${ }^{1}$ G. Parisi, ${ }^{2}$ and F. Ricci-Tersenghi ${ }^{2}$ \\ ${ }^{1}$ Université Paris-Sud, LPTMS, UMR8626, \\ Bât. 100, 91405 Orsay cedex, France \\ ${ }^{2}$ Dipartimento di Fisica, INFM-CNR SMC and INFN sez. di Roma1, \\ Università di Roma "La Sapienza", \\ P.le A. Moro 2, Roma 00185, Italy
}

(Dated: November 3, 2018)

\begin{abstract}
In this paper we study finite interaction range corrections to the mosaic picture of the glass transition as emerges from the study of the Kac limit of large interaction range for disordered models. To this aim we consider point to set correlation functions, or overlaps, in a one dimensional random energy model as a function of the range of interaction. In the Kac limit, the mosaic length defines a sharp first order transition separating a high overlap phase from a low overlap one. Correspondingly we find that overlap curves as a function of the window size and different finite interaction ranges cross roughly at the mosaic lenght. Nonetheless we find very slow convergence to the Kac limit and we discuss why this could be a problem for measuring the mosaic lenght in realistic models.

PACS numbers: 05.20.-y (Classical statistical mechanics), 64.70.Pf (Glass transitions), 75.10Nr (Spin-glass and other random models)
\end{abstract}




\section{INTRODUCTION}

The paradigm of 'random first order transition' or one-step replica symmetry breaking (1RSB) theory, provides an elegant framework to conceptualize the phenomenology of liquids approaching the glass transition [1]. Unfortunatly, this scenario is strongly based on meanfield models [2] and mean-field-like approximations to liquid theories [3] and cannot be taken literally in the application to real system. The main node that has to be untied to establish the 1RSB scenario as a convincing theory for real materials, is how mean-field theory should be adapted and modified to take into account the finite range of interactions. Though a fundamental theory of glassy systems in finite dimension is presently lacking, proposals have been made that modify minimally mean field scenario to take into account the finite interaction range. In ref. [2] Kirkpatrick, Thiurmialai and Wolynes developed a phenomenological theory, known as 'mosaic picture', where it is postulated the existence of a coherence length, that grows on lowering the temperature. Below that length the system behaves essentially as a mean-field glass, while it would cross-over to liquid behavior at larger scales. It results a theory where relaxation is dominated by activated processes stemming from the competition between interface tension and a bulk configurational entropy. The mosaic picture has been recently revived and deeply clarified by Biroli and Bouchaud [4], who showed that while usual (point-to-point) correlation functions are insensitive to the possible growth of the coherence mosaic length, it is possible to define different "point-to-set" correlation functions, able to reveal the growth of the mosaic length. In turn, the mosaic length has been related to the relaxation time of ordinary, time dependent correlation functions [5]. These papers prompted on one side numerical simulations on kinetically constrained glasses [6] and on realistic glassy models [7], on the other to theoretical calculations for models on trees and under the Kac limit [8]. These last models are the natural starting point for understanding the mosaic picture, since their local properties are well described by mean-field theory [9].

In [8] the study of point to set correlation function has allowed to derive a detailed picture relating the relaxation in the Mode Coupling regime for $T>T_{d}$ to the one in the mosaic regime for $T<T_{d}$. The calculation, supposedly exact, concern the behavior of disorderd glasses in the Kac limit. In order to understand its relevance for short range systems, it is necessary to study the properties of convergence to the Kac limit for finite interaction range. It has been found in ref. [7] that in standard Lennard-Jones supercooled liquids, the 
transition from high to small overlap as a function of the box size is much smoother than one would expect from the mosaic picture. This poses the question of what behavior one should expect when the range of interaction is not large.

In this paper, we address this question in a minimalistic finite dimensional model displaying 1RSB behavior in the Kac limit. The model is a one dimensional version of the Random Energy model [10] extensively studied in the context of stochastic models for reaction diffusion equations and evolving populations [11]. This has two main advantages: on one hand the Kac limit can be studied directly by probabilistic arguments, without having to resort to replicas or cavity techniques, on the other the model for finite interaction range can be studied exactly by transfer matrices.

A recent paper addresses the problem of finite range corrections to the mosaic picture in a related one dimensional XORSAT model [12]. That paper concerns the zero temperature limit, while we concentrate on finite temperature properties.

The organization of the paper is the following: in section [I] we define the model. Section 【iI is devoted to the definition of the point to set correlation we study. In section IV we discuss theoretical approaches to the computation of this quantity. In section $\nabla$ we discuss the results of exact computations with transfer matrices. Finally we draw our conclusions.

\section{THE MODEL}

In order to compare the behavior of finite range interaction systems with mean-field theories we need a model with variable interaction range which is well suited for numerical analysis. We decided to consider a 1D version of the Random Energy Model (REM) [10] introduced in the first of ref. [11]. This consists in a line of $m L$ Ising spins, divided in $L$ groups of $m$ spins such that only neighbouring groups of spins interact (thus leading to an interaction range of $2 m$ ).

For each group $i=1, \ldots, L$ we define a state variable $\sigma_{i}$ taking values $1, \ldots, 2^{m}$. In the variables $\left\{\sigma_{i}\right\}$ the interactions are restricted to nearest neighbors. The Hamiltonian of the system is

$$
H(\vec{\sigma})=\sum_{i=0}^{L-1} E_{i}\left(\sigma_{i}, \sigma_{i+1}\right) .
$$

For each link the $2^{2 m}$ interaction energies $E_{i}(\sigma, \tau)$ are quenched random variables extracted 
from a Gaussian distribution of zero mean and variance

$$
\overline{E^{2}}=m / 2 \text {. }
$$

We have considered fixed boundary conditions on the left side (in $i=0$ ), defining $\sigma_{0}=1$, and open boundary conditions on the right side (in $i=L$ ). In this way we minimize the computational effort needed to compute the free-energy $Z_{L}$, which is expressed as $Z_{L}=$ $\sum_{\sigma} Z_{L}(\sigma)$, where $Z_{L}(\sigma)$ is given by the recursion relation

$$
Z_{\ell+1}(\sigma)=\sum_{\tau=1}^{2^{m}} Z_{\ell}(\tau) e^{-\beta E_{\ell}(\tau, \sigma)},
$$

with $Z_{0}=1$ and $\beta=1 / T$. Computing $Z_{L}$ thus requires $\varnothing\left(L 2^{2 m}\right)$ operations.

In the $m \rightarrow \infty$ limit, the thermodynamics is simple: the correlations between the energy level implied by the one dimensional structure are negligible and, independently of $L$,

the free-energy coincides with the one of a REM with $2^{m L}$ states and energies distributed according to $P(E) \propto \exp \left(-E^{2} / m L\right)$ :

$$
F=\lim _{m \rightarrow \infty}-\frac{T}{m L} \log Z_{L}=\left\{\begin{array}{cc}
-\frac{\beta}{4}-T \log (2) & T>T_{c}, \\
-\sqrt{\log (2)} & T \leq T_{c}
\end{array}\right.
$$

with $T_{c}=(2 \sqrt{\log (2)})^{-1}$.

\section{THE OBSERVABLES}

Here we define the correlation functions of interest, allowing us to detect a growing static length. These are built with the aid of a suitably chosen reference configuration $\left\{\sigma_{i}^{*}\right\}_{i=1, \ldots, L}$, to which one fixes the system outside a window with $\ell$ sites located around the center of the system. For convenience we renumber $1, \ldots, \ell$ the sites in the central window. Inside the window the system is at thermal equilibrium. We investigate the correlation among typical in-window configurations $\vec{\sigma}$ with $\vec{\sigma}^{*}$ to see whether a characteristic length exist $\ell_{c}$ such that for window sizes $\ell<\ell_{c}, \vec{\sigma} \simeq \vec{\sigma}^{*}$ inside the window, while, for $\ell>\ell_{c}, \vec{\sigma}$ and $\vec{\sigma}^{*}$ are uncorrelated.

As detailed in the following, in order to sharpen the transition from correlated to uncorrelated behavior we decided to fix the reference configuration $\vec{\sigma}^{*}$ always to the ground state. 
We then study the thermodynamics of a system which is fixed to the reference configuration outside a window of size $\ell$ :

$$
\sigma_{i}=\sigma_{i}^{*} \quad \forall i<1 \text { and } \forall i>\ell
$$

The system has then fixed boundaries and $\ell$ free variables, $\left\{\sigma_{i}\right\}_{i=1, \ldots, \ell}$. Within the window, we can define its overlap with respect to the reference configuration as

$$
q\left(\vec{\sigma}, \vec{\sigma}^{*}\right) \equiv \frac{1}{\ell} \sum_{i=1}^{\ell} \delta\left(\sigma_{i}, \sigma_{i}^{*}\right) .
$$

Notice that our point-to-set correlation function differs from the one defined in [4] and used subsequently which consists in choosing $\sigma^{*}$ as a configuration thermalized at temperature $T$.

We need some observable estimating the similarity of the typical configuration with respect to the reference one, and to this end we introduce the following two quantities:

$$
\begin{aligned}
p_{0}(\ell, \beta) & \equiv \frac{e^{-\beta \sum_{i=0}^{\ell} E_{i}\left(\sigma_{i}^{*}, \sigma_{i+1}^{*}\right)}}{\sum_{\left\{\sigma_{i}\right\}_{i=1}^{\ell}} e^{-\beta \sum_{i=0}^{\ell} E_{i}\left(\sigma_{i}, \sigma_{i+1}\right)}}, \\
q_{0}(\ell, \beta) & \equiv \frac{\sum_{\left\{\sigma_{i}\right\}_{i=1}^{\ell}} q\left(\vec{\sigma}, \vec{\sigma}^{*}\right) e^{-\beta \sum_{i=0}^{\ell} E_{i}\left(\sigma_{i}, \sigma_{i+1}\right)}}{\sum_{\left\{\sigma_{i}\right\}_{i=1}^{\ell}} e^{-\beta \sum_{i=0}^{\ell} E_{i}\left(\sigma_{i}, \sigma_{i+1}\right)}},
\end{aligned}
$$

where the denominator is the "window partition function". The first quantity, $p_{0}$, is the relative weight of the reference configuration in the window partition function computed at inverse temperature $\beta$, while the second quantity, $q_{0}$, is the mean overlap with the reference configuration. Both quantities still depend on the quenched disorder and we compute their typical values by $\log \left(p_{\text {typ }}\right) \equiv \overline{\log \left(p_{0}\right)}$ and $\log \left(q_{\text {typ }}\right) \equiv \overline{\log \left(q_{0}\right)}$, where the overline stands for the average over the quenched disorder. We expect $\log \left(p_{0}\right)$ and $\log \left(q_{0}\right)$ to be self-averaging, since their are related to free-energy differences.

\section{THEORETICAL ANALYSIS}

In this section we address the problem of an analytic computation of the correlation functions. We will first study exactly the asymptotic long range limit $m \rightarrow \infty$. After that we will address the problem of finite $m$ effects, that our numerical analysis below reveals to be very large. 


\section{A. The correlation functions for $m \rightarrow \infty$}

The infinite $m$ limit can be understood since in this limit the correlations between the energy level due to the one-dimensional structure of the model become negligible. In this case, using this independence approximation, we see that, besides the state $\vec{\sigma}^{*}$ of energy $E^{*}$, the window has $2^{m \ell}-1$ states with energies distributed according to $P_{\ell}(E) \propto e^{-E^{2} / m(\ell+1)}$ (there are $\ell$ sites and $\ell+1$ links!). So that the average density of states is

$$
\mathcal{N}(E) \sim 2^{m \ell} e^{-E^{2} / m(\ell+1)}+\delta_{E, E^{*}}
$$

and the microcanonical entropy per link (divided by $m$ ) as a function of the link energy $\epsilon=E / m(\ell+1)$ is

$$
S_{\ell}(\epsilon)=\left\{\begin{array}{cl}
-\epsilon^{2}+\frac{1}{1+1 / \ell} \log (2) & |\epsilon|<\sqrt{\frac{1}{1+1 / \ell} \log (2)} \\
0 & \text { otherwise }
\end{array}\right.
$$

From this function the canonical thermodynamics can be derived. Before doing that, few comments are in order: (a) The constrained entropy is reduced by a constant term with respect to the unconstrained case, given by the above formula with $\ell=\infty$ (see Fig. 1). (b) The choice of the reference configuration $\vec{\sigma}^{*}$ as the ground state has no effect on the other states: the same entropy would be obtained for different choices of $\sigma^{*}$. Of course the window thermodynamics and correlations would depend on the energy of $\vec{\sigma}^{*}$.

If the state $\vec{\sigma}^{*}$ was absent, the free-energy per link would read

$$
\tilde{f}(\beta, \ell)=\left\{\begin{array}{cl}
-\frac{\beta}{4}-\frac{T}{1+1 / \ell} \log (2) & T>T_{c} \sqrt{1+1 / \ell} \\
-\sqrt{\frac{1}{1+1 / \ell} \log (2)} & T \leq T_{c} \sqrt{1+1 / \ell} .
\end{array}\right.
$$

Including the state $\vec{\sigma}^{*}$ one therefore has

$$
f(\beta, \ell)=\min \left\{\epsilon^{*}, \tilde{f}(\beta, \ell)\right\},
$$

with $\epsilon^{*}=-\sqrt{\log (2)}$, that is the ground state energy of the $\ell=\infty$ system. When the two terms in Eq.(12) are equal, a first order transition takes place (see Fig. 11) at inverse temperature

$$
\beta_{c}(\ell)=2 \sqrt{\log (2)}\left(1-\frac{1}{\sqrt{\ell+1}}\right)=\beta_{c}\left(1-\frac{1}{\sqrt{\ell+1}}\right),
$$

which in turns defines a temperature dependent critical length

$$
\ell_{c}(\beta)=\frac{\beta\left(2 \beta_{c}-\beta\right)}{\left(\beta_{c}-\beta\right)^{2}},
$$




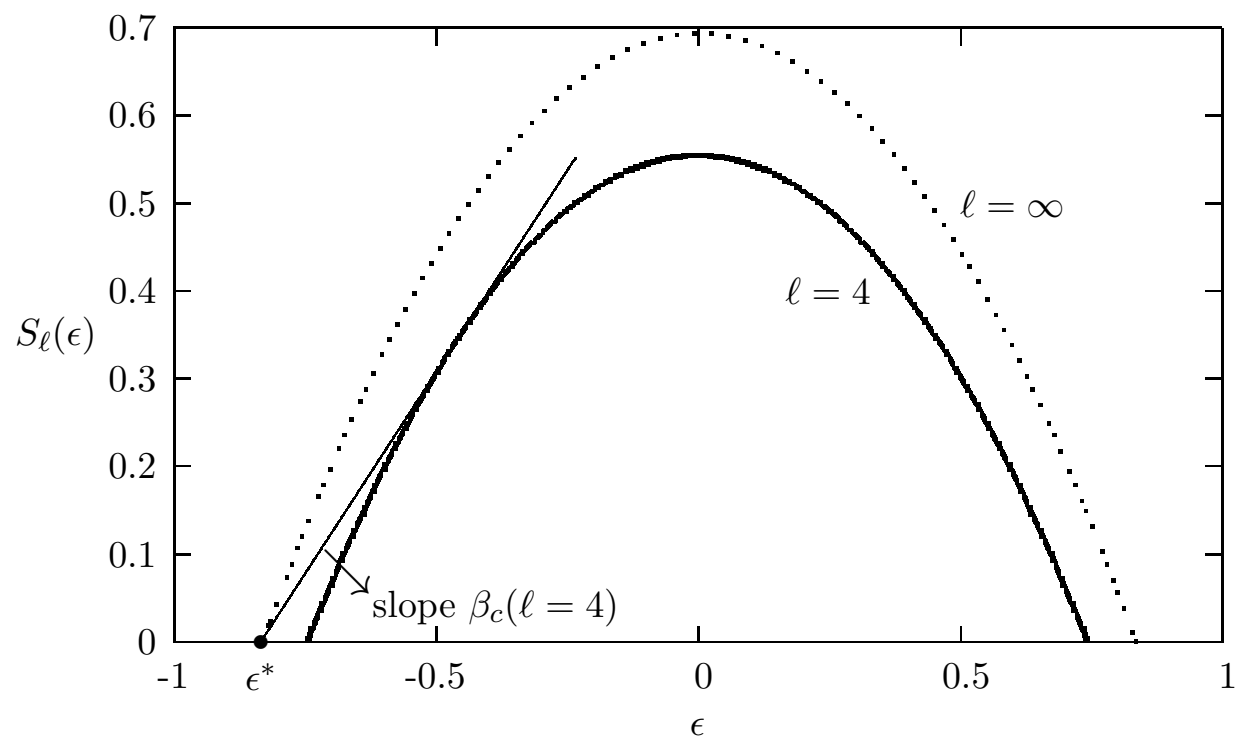

FIG. 1: Microcanonical entropy for the full system $(\ell=\infty$, dotted line) and for a window of size $\ell=4$ (thick line). A system prepared in the ground state of the full system (big dot) and constrained in a window of size $\ell=4$ makes a first order transition at inverse temperature $\beta_{c}(\ell=4)$ (the slope of the thin line).

separating the confined regime $\ell<\ell_{c}$ where $p_{t y p}=q_{t y p}=1$ from the deconfined regime $\ell>\ell_{c}$ where $p_{t y p}=q_{t y p}=0$.

The size of the critical window diverges as expected at the critical temperature, where the configurationl entropy vanishes. We find that this critical length is quardatic in the inverse of $T-T_{c}$; had we chosen the reference state $\vec{\sigma}^{*}$ with a different rule, the result would have been different. For example a direct calculation shows that choosing $\vec{\sigma}^{*}$ with Boltzmann probability at temperature $T$ implies a linear critical length in $1 /\left(T-T_{c}\right)$.

We can understand better the structure of the excitations studying the window free-energy as a function of the overlap $q$, i.e. the free-energy of configurations that do not coincide with $\vec{\sigma}^{*}$ on exaclty $d$ sites among the $\ell$ of the window, with $q=1-d / \ell$. For simplicity we can consider the contribution of "one bubble configurations" where all the $d$ sites in question are contiguous. We show below that configurations with more that one bubble are exponentially unprobable for large $m$ values.

For large $m$ the dominant contribution to the free-energy per link $f(\beta, \ell, d)=$ 


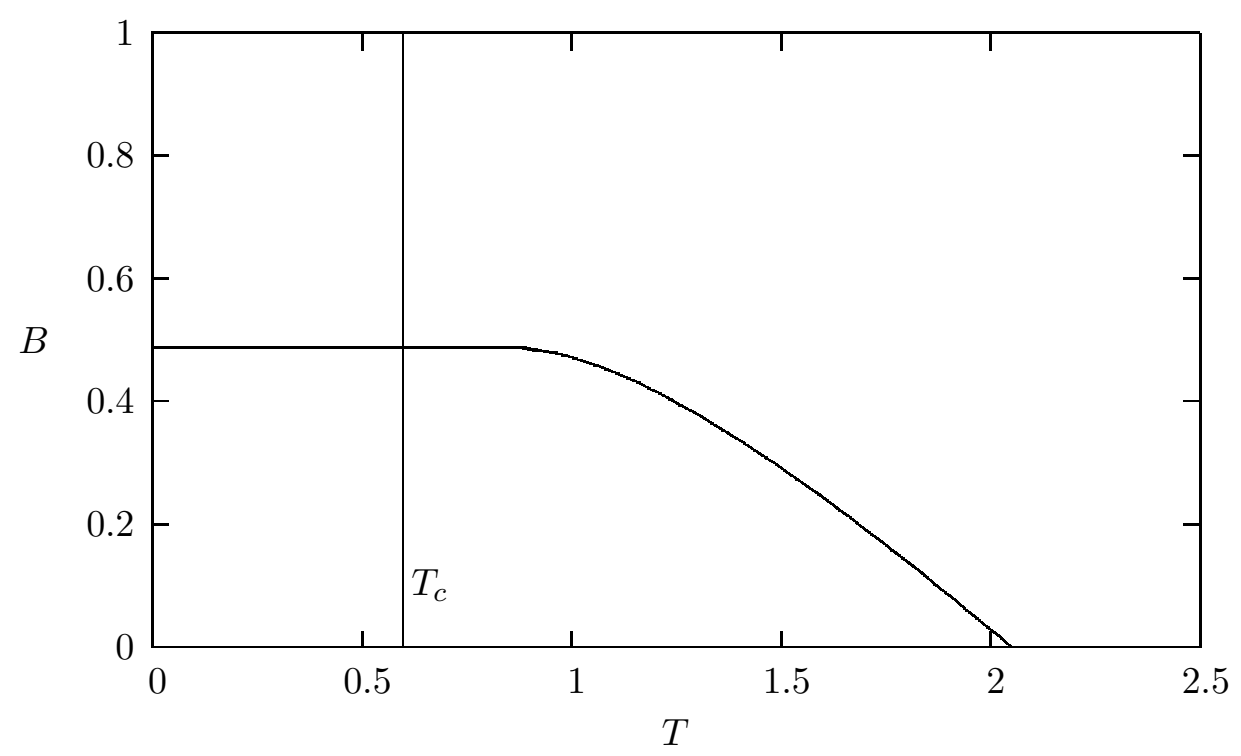

FIG. 2: The free-energy barrier to relax from the ground state $\vec{\sigma}^{*}$.

$\lim _{m \rightarrow \infty} F / m(\ell+1)$ is independent of the position of the bubble and reads, for $d=1, \ldots, \ell$ :

$$
f(\beta, \ell, d)=\left\{\begin{array}{cl}
-\frac{\ell-d}{\ell+1} \sqrt{\log (2)}-\frac{d+1}{\ell+1}\left(\frac{\beta}{4}+\frac{T}{1+1 / d} \log (2)\right) & T>T_{c} \sqrt{1+1 / d}, \\
-\frac{\ell-d}{\ell+1} \sqrt{\log (2)}-\frac{d+1}{\ell+1} \sqrt{\frac{1}{1+1 / d} \log (2)} & T \leq T_{c} \sqrt{1+1 / d} .
\end{array}\right.
$$

For $d=0$ the free-energy is simply given by $f(\beta, \ell, 0)=\epsilon^{*}$. As one can explicitly see, $f$ is monotonically decreasing in $d$ : the completely open configuration is always the most favoured among the ones with $d \geq 1$. Notice that at low temperature $f(\beta, \ell, 1)>f(\beta, \ell, 0)$ and the difference $B(\beta)=(l+1)[f(\beta, \ell, 1)-f(\beta, \ell, 0)]$ can be interpreted as a relaxation free-energy barrier for a system prepared in the ground state $\vec{\sigma}^{*}$. The barrier $B(\beta)$, plotted in Fig. 2, is $\ell$-independent and vanishes at a temperature $T=T_{c} /(1-1 / \sqrt{2})$.

If we remove the assumption of considering only one-bubble configurations, the freeenergy in Eq.(15) becomes

$$
f(\beta, \ell, d)=\min _{b \in\{1, d / 2\}} f(\beta, \ell, d, b)
$$

where $f(\beta, \ell, d, b)$ is the free-energy of configurations differing in $d$ variables from $\vec{\sigma}^{*}$ and having $b$ bubbles, given by the following expression in the large $m$ limit:

$$
f(\beta, \ell, d, b)=\left\{\begin{array}{cl}
-\frac{\ell+1-d-b}{\ell+1} \sqrt{\log (2)}-\frac{d+b}{\ell+1}\left(\frac{\beta}{4}+\frac{T}{1+b / d} \log (2)\right) & T>T_{c} \sqrt{1+b / d}, \\
-\frac{\ell+1-d-b}{\ell+1} \sqrt{\log (2)}-\frac{d+b}{\ell+1} \sqrt{\frac{1}{1+b / d} \log (2)} & T \leq T_{c} \sqrt{1+b / d} .
\end{array}\right.
$$


It is easy to verify that the minimum in Eq.(16) is always achieved in $b=1$, i.e. on onebubble configurations. Multi-bubble configurations can only modify the corrections to the leading behavior in $m$.

\section{B. Analysis of the Ground State}

We would like to present here some attempts to take into account finite $m$ contributions. Corrections to the asymptotic result have two sources: the correlations between the levels and sample-to-sample fluctuations. Though we were not able to deal with the former, we could analyze some of the latter.

Actually we derive some analytical results under 2 main approximations, namely (i) energy levels are basically treated as uncorrelated and (ii) the energy of the reference configuration (the ground state energy $E^{*}$ ) is considered to be evenly distributed among the links, each one having a local energy $m \epsilon^{*}=E^{*} / L$ (please note that the entire system is made of $L$ links, while the window had $\ell+1$ links). We will see below that numerical evidence shows that this is the case not too close to the boundaries $i=0$ and $i=L$.

The distribution of $\epsilon^{*}$ is known for $L=1$, since in that case $m \epsilon^{*}$ corresponds to the minimum among $2^{m}$ independent random Gaussian variables of variance $m / 2$, that is

$$
\epsilon^{*}(L=1) \stackrel{\mathrm{d}}{=}-\sqrt{\log (2)}+\frac{\log (m)+\log (4 \pi \log (2))+2 X}{4 m \sqrt{\log (2)}}+\varnothing\left(\frac{\log (m)}{m^{2}}\right),
$$

where $X$ is a Gumbel distributed variable, i.e. $\mathbb{P}(X>x)=e^{-e^{x}}$. Similarly a closed formula can be obtained for $L=2$ which corresponds to a two level GREM. Unfortunately as soon as $L>2$ there are no exact results on the ground state energy of the model. In this case an analitical upper bound can be simply constructed by the following greedy algorithm: given that $\sigma_{0}$ is fixed, assign $\sigma_{1}$ to the value minimizing $E\left(\sigma_{0}, \sigma_{1}\right)$ and repeat the procedure recursively on the next variable; at each step the link energy has the same probability distribution as $m \epsilon^{*}(L=1)$, and so the global ground state energy satisfies $E^{*}(m, L) \leq m L \epsilon^{*}(L=1)$.

Our numerical data suggest this bound to be tight at the leading order in $m$ for any value of $L$. More precisely, we find numerically that the mean ground state energy can be very well fitted, for large values of $L$, by the following formula:

$$
\frac{E^{*}(m, L)}{m L} \simeq-\sqrt{\log (2)}+\frac{A}{m^{3 / 2}}+\frac{B}{m^{1 / 2} L},
$$




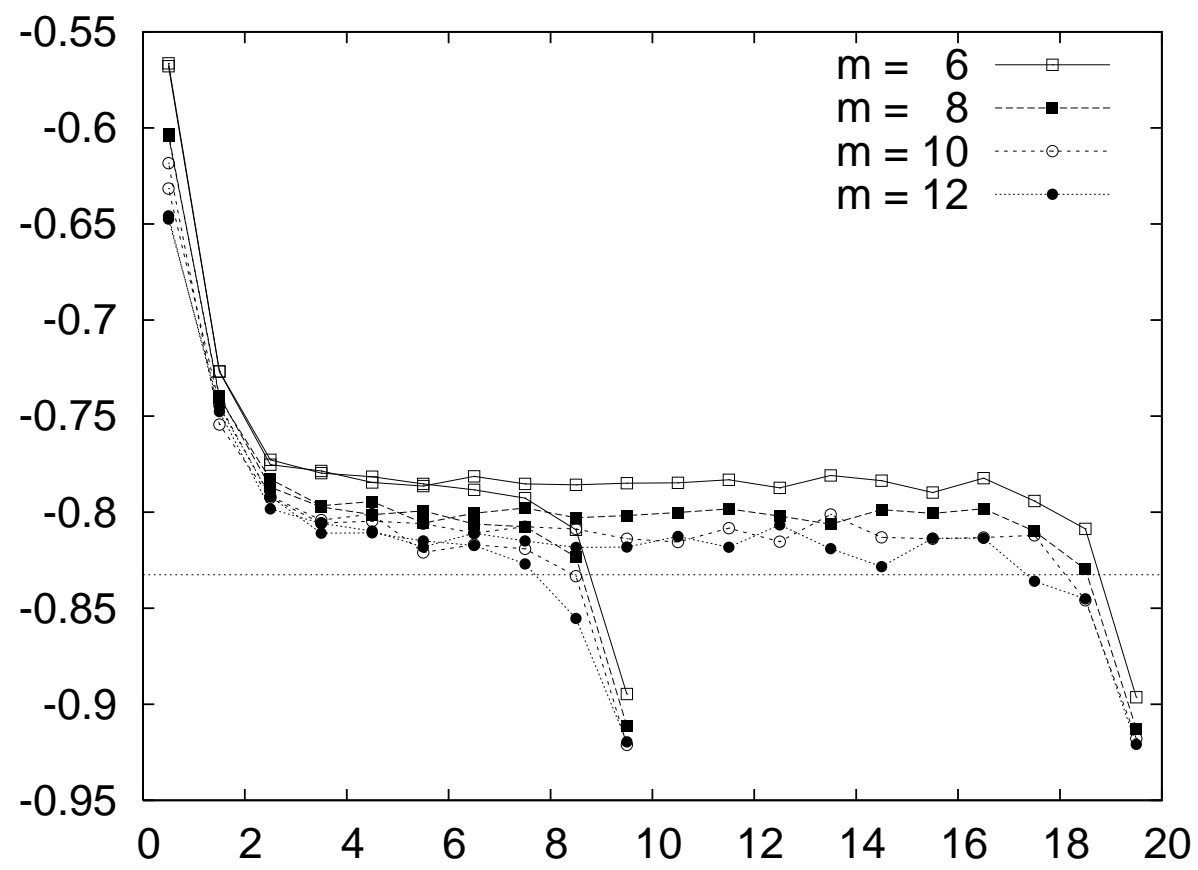

FIG. 3: The mean value of the ground state link energy as a function of the position in the system for $L=10$ and $L=20$. On the left boundary the configuration is fixed, while on the right end the system is free. The horizontal line is $-\sqrt{\log (2)}$.

with $A \sim 0.7$ and $B \sim 0.4$. This behavior clearly shows that the convergence to the asymptotic intensive energy, $-\sqrt{\log (2)}$, becomes faster increasing $L$ : for $L=1$ corrections are $\varnothing\left(\frac{\log (m)}{m}\right)$ and they become $\varnothing\left(m^{-3 / 2}\right)$ in the $L \rightarrow \infty$ limit. We see from Fig. 3 that already for $L \sim 10$, not too close to the boundaries, ground states link energies are independent of $L$, and their numerical values are well represented by the previous formula with $L=\infty$. Obviuosly, given the values of $m$ we can study, formula (19) has to be taken as an empirical interpolating function. We find from our data that in ground state configurations, link energies have very small sample-to-sample fluctuations, which decrease for larger $m$ values: for this reason considering only mean values for the link energies is a good approximation. In order to minimize finite $L$ effects and have a homogeneous ground state inside the window we find that it was enough to consider sistem sizes $L=\ell+20$, i.e. 10 sites between the window and system boundaries.

Once understood the ground state structure, let us now turn to the estimate of the window correlation functions. 


\section{Finite $m$ estimates of the correlation functions}

Under the assumptions stated above, the weight, in the window partition function, of all the configurations differing in $d$ variables with respect to $\vec{\sigma}^{*}$ is given by

$$
Z_{d}=(\ell+1-d)\left(2^{m}-1\right)^{d} \frac{\int_{(d+1) m \epsilon^{*}}^{\infty} d z e^{-\beta z-\frac{z^{2}}{m(d+1)}}}{\int_{(d+1) m \epsilon^{*}}^{\infty} d z e^{-\frac{z^{2}}{m(d+1)}}} e^{-\beta(\ell-d) m \epsilon^{*}}
$$

where the first term gives the number of ways to place a bubble of size $d$ in a window of size $\ell$, the second term counts the number of configurations of the $d$ variables which have to differ from $\vec{\sigma}^{*}$, the fraction is the average of $e^{-\beta H}$ over the p.d.f. of the energies of the bubble (it is the sum of $d+1$ Gaussian variables of variance $m / 2$, bounded from below by the ground state energy, $\left.(d+1) m \epsilon^{*}\right)$ and the last term is given by the $l-d$ links having the ground state energy. In equation (20) we have that $d \in\{1, \ldots, \ell\}$, while the weight of the ground state is given by $Z_{0}=\exp \left(-\beta E^{*}\right)=\exp \left(-\beta(\ell+1) m \epsilon^{*}\right)$. We do not write explicitly the dependence of $Z_{d}$ on $\beta, \ell, m$ and $\epsilon^{*}$ in order to keep the notation light.

$Z_{d}$ is an annealed approximation for the window partition function at a fixed distance from the ground state. Still, the fact that we keep the dependence on $\epsilon^{*}$ explicit is important

in order to control some fluctuations: e.g. both $\log \left(p_{0}\right)$ and $\log \left(q_{0}\right)$ are given by free-energy differences, where the dependence on $\epsilon^{*}$ is partially canceled out, and their average over $\epsilon^{*}$ can be done without any approximation. The two observables we are interested in are indeed given by

$$
p_{0}=\frac{Z_{0}}{\sum_{d=0}^{\ell} Z_{d}}, \quad q_{0}=\frac{\sum_{d=0}^{\ell}(1-d / \ell) Z_{d}}{\sum_{d=0}^{\ell} Z_{d}},
$$

and can be easily computed by evaluating numerically the integrals in the definition of $Z_{d}$, once the p.d.f. of $\epsilon^{*}$ is known. We have measured numerically such a distribution, but once we plugged it into Eq.(20) we discovered that the observables we are interested in ( $p_{t y p}$ and $q_{\text {typ }}$ ) mainly depend on the mean of $\epsilon^{*}$, being such a distribution very narrow. Moreover we are mostly interested in the dependence of these observables on $m$ in order to understand the approach to the $m \rightarrow \infty$ limit, and the average of $\epsilon^{*}$ carries the largest dependence on $m$.

For these reasons the analytical curves we are going to compare with numerical data in the next section have been obtained using a non-fluctuating value for $\epsilon^{*}$, give by Eq.(19) that is $\epsilon^{*}=-\sqrt{\log (2)}+0.726 / m^{3 / 2}$. As we show below, this dependence on the interaction 
range is already enough to produce strong finite $m$ effects. Remind that, in the $m \rightarrow \infty$ limit, the logarithm of $Z_{d}$ is given by the free-energy in Eq.(15), and both $p_{t y p}$ and $q_{t y p}$ should drop from 1 to 0 when $\ell$ crosses the value of $\ell_{c}(\beta)$ given by Eq.(14).

\section{NUMERICAL RESULTS}

The aim of this section is to compute numerically the above defined critical length scale for the 1D random energy model. The numerical experiment we have performed consists in:

1. computing the ground state of a system of size $L$;

2. fixing the ground state configuration outside a window of size $\ell$;

3. computing $q_{t y p}$ and $p_{t y p}$ in order to see whether there is a first order transition in these quantities varying the window size $\ell$.

In the $m \rightarrow \infty$ limit we expect such a transition when the window size crosses the value $\ell_{c}(\beta)$ given in Eq.(14). For finite values of $m$ the system cannot have any transition (it is one-dimensional), but still the crossover may be very sharp. Our main interest is in understanding how much the behavior of finite $m$ systems resembles the mean-field (i.e. $m \rightarrow \infty)$ limit and how fast is the convergence.

As explained in the previous section, we take the size of the system $L$ larger than the size of the window in order to avoid boundary effects; that is, to all practical purposes we are working in the $L \rightarrow \infty$ limit.

Thanks to the one-dimensional topology all the experiments can be done exactly by tranfer matrix methods. Unfortunately for each link we have a different random matrix with $2^{m} \times 2^{m}$ entries; for this reason we are forced to small values of $m$ (actually we use $m=6,8,10,12)$. Please note that these $m$ values are not so small: the number of degrees of freedom per region $\left(2^{m}\right)$ is comparable or even larger than the number of particles within a typical region studied in realistic models of glassy systems [7]. Since we are interested in computing the free-energy at a given value of the overlap with the reference configuration, the transfer matrix computation is slightly more complicated and requires a total time of order $\mathcal{O}\left(\ell^{2} 2^{2 m}\right)$. The average over the disorder is done with at least 1000 samples for any $m$ value.

We are going to present results for temperature $T=0.8$, which is a very reasonable value (in the $m \rightarrow \infty$ limit the critical temperature is $T_{c}=0.60056 \ldots$ ), since the critical window 

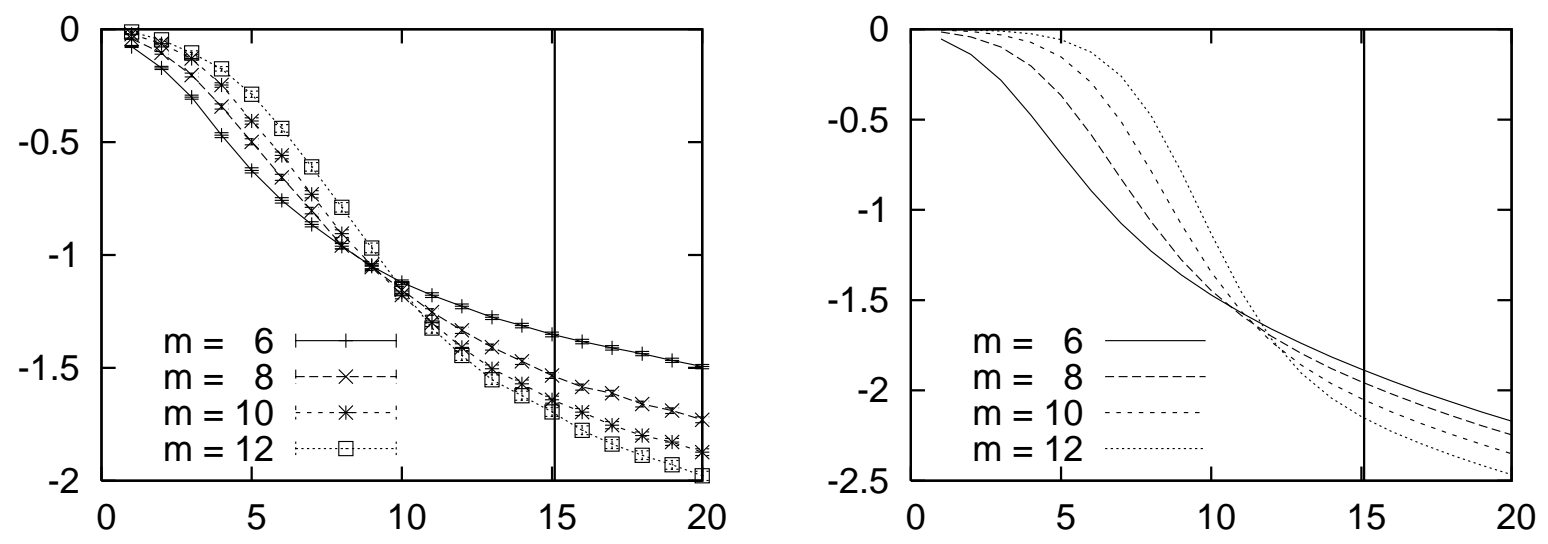

FIG. 4: The overlap $q_{t y p}$ as a function of $\ell$ at $T=0.8$. Left panel: numerical values obtained throught the transfer matrix algorithm for various values of $m$. Right panel: analytic curves obtained through the approximations discussed in the text. The vertical lines marks the value of $\ell_{c}$ in the $m \rightarrow \infty$ limit.
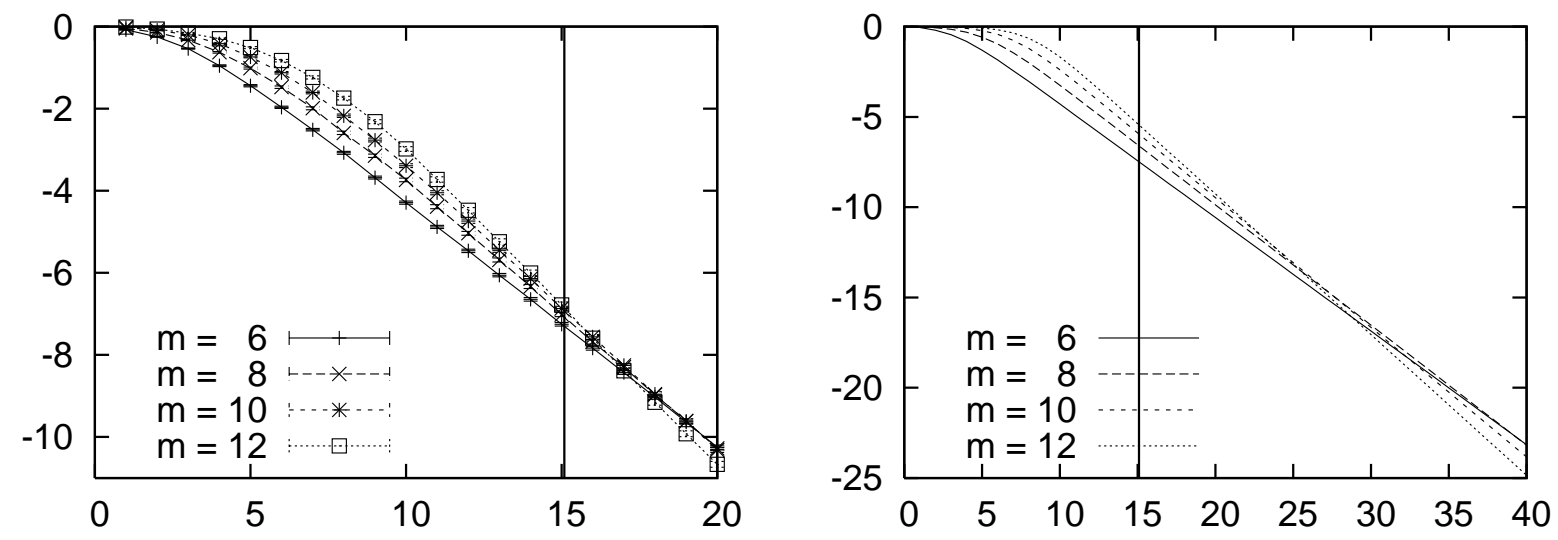

FIG. 5: Same as figure 4 for the quantity $p_{t y p}$.

size is $\ell_{c}(T=0.8)=15.09$.

In Fig. 4 and 5 we show respectiely $\log \left(q_{\text {typ }}\right)$ and $\log \left(p_{\text {typ }}\right)$ as a a function of $\ell$. Left panels reports data from exact numerical computations, while right panels show the outcome of the analytical approximated analysis. The vertical line is the critical window size $\ell_{c}$.

Some comments are in order. The behavior of all the curves for different $m$ hints at a first order transition for $m \rightarrow \infty$ separating a high overlap region at small $\ell$ from a zero overlap region at large $\ell$. This behavior is in agreement with the prediction of the mosaic theory, however, the convergence is very slow! Indeed so slow, that it does not allow an 
estimate of the speed of convergence. The crossing point of numerical data for $\log \left(q_{\text {typ }}\right)$ is around $\ell=10$, well below the predicted $\ell_{c}=15.09$. In principle one could argue that the one-dimensional model may have a first order transition at a lower value of $\ell_{c}$, but the crossing point of numerical data for $\log \left(p_{\text {typ }}\right)$, taking place around $\ell=17$, suggests that the crossing point is strongly dependent on $m$ and converges for $m \rightarrow \infty$ somewhere between 10 and 17 (we are assuming that both $q_{\text {typ }}$ and $p_{\text {typ }}$ have a jump at the same value of $\ell$ for $m \rightarrow \infty)$.

Still more evident indications of strong finite $m$ effects come from the analytical curves (see right panels of Fig. 4 and Fig. [5): these have been computed from Eq.(20) and Eq.(21) with $\epsilon^{*}=-\sqrt{\log (2)}+0.726 m^{-3 / 2}$, see Eq.(19), which is the best interpolation for the ground state energy in the window, far from the boundaries. Although these curves have been obtained under some approximations, they look qualitatively very similar to the exact numerical data, and also quantitatively are not far from the data. For the analytical curves we know that they have a jump in $\ell=\ell_{c}$ in the $m \rightarrow \infty$ limit, still for the present values of $m$ they show a crossing point quite far from $\ell_{c}$.

Moreover the value of the overlap at the crossing point may be very small, depending on the overlap one is looking at (see e.g. the value of $p_{t y p}$ at the crossing point). For this reason may be very difficult to locate the crossing point (remember that our model has a very strong random first order transition in the $m \rightarrow \infty$ limit, and most probably things work even worst in more realistic models!).

We remark that simulating the model for a single value of $m$ it would be difficult to claim any agreement with the 1RSB theory of glasses and the mosaic state: it is comparing different values on the interaction range $m$ that the agreement becomes apparent. One could argument that one dimension is the worst possibility to observe any behavior reminiscent of a phase transition and in higher dimension the situation could be more favourable to the theory. Recent simulations of more realistic binary Lennard-Jones mixtures [7] however, failed to identify a sharp mosaic length.

In order to understand better why $q_{t y p}$ has such strong finite $m$ corrections and show an effective crossing point at window sizes smaller than $\ell_{c}$, we have studied the window free energy as a function of the overlap with respect to the ground state. We show in Fig. 6] such a free-energy for $T=0.8, \ell=10$ and many values of $m$ in order to study the dependence on $m$. We see that, increasing the value of $m$, all the curves $f(q)$ tends to decrease, but 


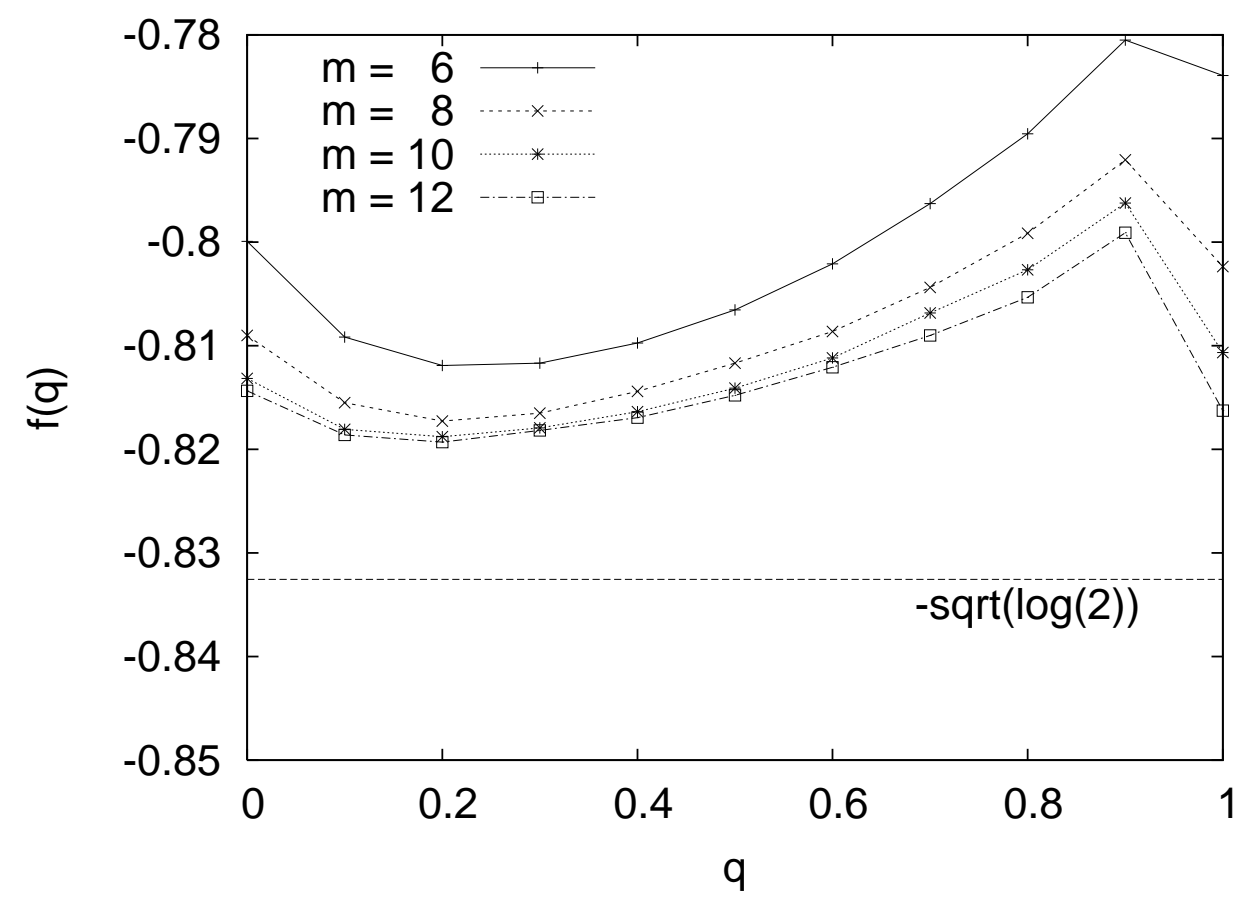

FIG. 6: Window free-energy for $\ell=10$ and $T=0.8$ as a function of the overlap with respect to the ground state configuration.

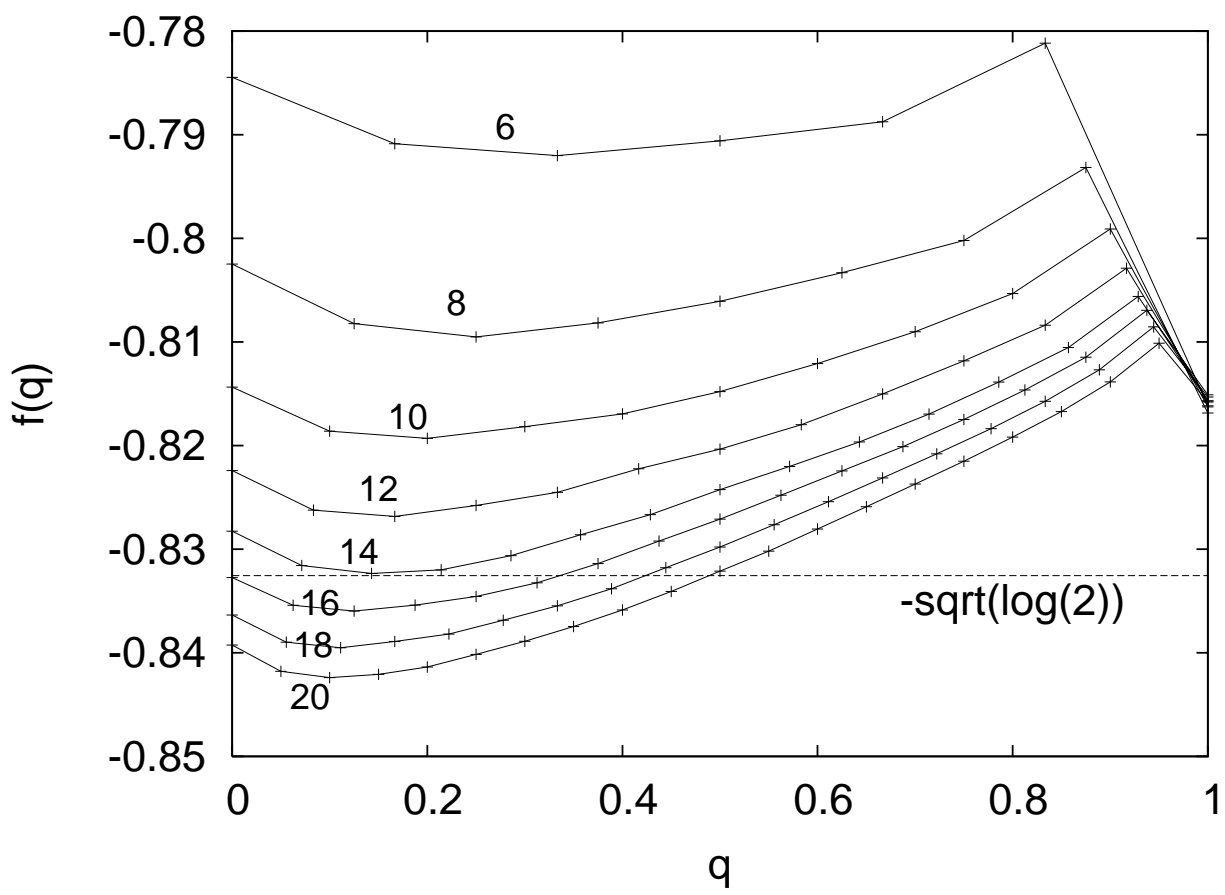

FIG. 7: Window free-energy for $T=0.8, m=12$ and many different values of $\ell$, from 6 (top) to 20 (bottom). 
corrections to the $m \rightarrow \infty$ limit are clearly larger for $f(q=1)$ than for the rest of the curve. Please note that $f(q=1)$ corresponds to the ground state energy, that converges in the $m \rightarrow \infty$ limit to $-\sqrt{\log (2)}$ (represented by the horizontal line in the plot). The different convergence rate for different $q$ values can be understood also from the analytical computation in the previous Section; indeed in the expression for $Z_{d}$, see Eq.(20), larger corrections are for small $d$ values (corresponding to larger $q$ ).

A discrepancy with respect to the analytical computation, is that the free-energy presents a minimum at a positive value of the overlap, while in the $m \rightarrow \infty$ limit we expect the minimum to be in $q=0$. This may be one more effect of the slow convergence to the mean-field limit.

In the tentative of extrapolating the numerical results to the $m \rightarrow \infty$ limit, we have fitted $f(q)$ data at fixed $q$, finding that the limit of $f(q=1)$ is always compatible with $-\sqrt{\log (2)}$, while for $q<1$ the asymptotic value of $f(q)$ is quite close to that computed numerically with $m=12$, especially close to the minimum of $f(q)$.

In Fig. 7 we show the free-energy $f(q)$ for $m=12$ (which is very close to the $m \rightarrow \infty$ value in the low $q$ region) for many $\ell$ values, ranging from 6 to 20 (top to bottom). The apparent first order phase transition between the $q=1$ and the small overlap regimes is taking place between $\ell=9$ and $\ell=10$ when the minimum goes below $f(q=1)[13]$, consistently to what we observe in the left panel of Fig. 4. Nonetheless, in the $m \rightarrow \infty$ we expect the transition to take place when the minimum goes below the value $-\sqrt{\log (2)}$, and we see from Fig. 7 that this happens around $\ell=14$, much closer to the predicted $\ell_{c}(T=0.8)=15.09$.

\section{CONCLUSIONS}

The scope of this paper is to study the properties of convergence to the mosaic picture in models with larger and larger interaction ranges. We showed that, as it should be expected, the behavior of point to set correlations approach the behavior predicted by the mosaic picture for large interaction range. The numerical evidence in favor of that comes from a differential analysis comparing the behavior for different values of the interaction range $m$. Curves at single values of $m$ do not allow to distinguish mosaic behavior from a single state picture where the point to set correlation exhibit a smooth behavior as a function of $\ell$. This is unfortunate as it indicates that it could be difficult to find confirmations or disprovals of 
the mosaic picture in realistic glass former models on the basis of the behavior of point to set correlations.

Some papers have recently addressed the study of point-to-set functions in non disordered models. Amazingly, the model where the mosaic predictions seems to fit better the data is a kinetically constrained model considered in [6] where a step like behavior of the overlap as a function of the window size is observed. Conversely, for a Lennard-Jones binary mixture, though it is observed a characteristic length growing with temperature, no step behavior is seen. We remark on this purpose that in our data it would been difficult to decide in favor of the mosaic picture on the basis of a single value of $m$. It is only comparing different values of $m$ that evidence for the first order jump has been obtained.

Moreover the convergence to the large $m$ limit is rather slow: finite $m$ curves are very smooth and show no precursor of the asymptotic step-like behavior. Ref. [12] studied a similar 1d model with finite interaction range, namely a XORSAT model. The main difference with respect to our study is that the model studied in ref. [12] possesses zero-energy ground states and it has been studied only at zero temperature. Despite these differences also in ref. [12] large finite-range effects have been found.

The main effect that we have seen in the model studied here is that a rather sharp transition takes place at a finite temperature $\beta_{c}(\ell)$ between a single low-energy ground state (i.e. of zero complexity) and a set of higher free-energy states (with positive complexity) in a way more or less similar to the mosaic picture. However this transition is plagued by large fluctuations mainly due to the energy of the ground state, which plays a fundamental role in determining the critical temperature: the final effect being a sizable smoothing of the random first order phase transition at finite value of the interaction range $m$.

The conclusions reached in this work suggest that the direct observation of the phase transition predicted within the mosaic theory may be rather difficult in realistic models, where the interaction range cannot be made very large. A smarter approach for the identification of such a transition is likely needed.

[1] See M. Mézard, Physica A 306, 25 (2002) for a recent review.

[2] T.R. Kirkpatrick and P. G. Wolynes, Phys. Rev. B 368552 (1987); T.R. Kirkpatrick, D. 
Thirumalai, and P.G. Wolynes, Phys. Rev. A 401045 (1989).

[3] M. Mézard and G. Parisi, Phys. Rev. Lett. 82, 747 (1999).

[4] J.-P. Bouchaud and G. Biroli, J. Chem. Phys. 121, 7347 (2004).

[5] A. Montanari and G. Semerjian, J. Stat. Phys. 125, 23 (2006).

[6] R.L. Jack and J.P. Garrahan, J. Chem. Phys. 123, 164508 (2005).

[7] A. Cavagna, T.S. Grigera, P. Verrocchio, Phys. Rev. Lett. 98, 187801 (2007).

[8] S. Franz and A. Montanari, J. Phys. A: Math. Gen. 40, F251 (2007).

[9] S. Franz and F.L. Toninelli, Phys. Rev. Lett. 92, 030602 (2004); J. Phys. A: Math. Gen. 37, 7433 (2004); J. Stat. Mech., P01008 (2005).

[10] B. Derrida, Phys. Rev. Lett. 45, 79 (1980).

[11] E. Brunet and B. Derrida, Phys. Rev. E 56, 2597 (1997); J. Stat. Phys. 103, 269 (2001); Phys. Rev. E 70, 016106 (2004). E. Brunet, B. Derrida, A. H. Mueller, S. Munier, Phys. Rev. E 73, 056126 (2006); Phys. Rev. E 76, 041104 (2007).

[12] A. Montanari and A. Sinton, A simple one dimensional glassy Kac model, preprint arXiv:0705.0054.

[13] Please note also that $f(q=1)$ does not depend on the window size $\ell$, confirming that the ground state in the window is insensitive to the boundaries. 